\title{
Dosimetric quantities and effective dose in medical imaging: a summary for medical doctors
}

\author{
Eliseo Vano ${ }^{1 *}$, Guy Frija ${ }^{2}$, Reinhard Loose ${ }^{3}$, Graciano Paulo ${ }^{4}$, Efstathios Efstathopoulos ${ }^{5}$, Claudio Granata ${ }^{6}$ and \\ Jonas Andersson ${ }^{7}$ on behalf of the European Society of Radiology (ESR)
}

\begin{abstract}
This review presents basic information on the dosimetric quantities used in medical imaging for reporting patient doses and establishing diagnostic reference levels. The proper use of the radiation protection quantity "effective dose" to compare doses delivered by different radiological procedures and different imaging modalities with its uncertainties and limitations, is summarised. The estimates of population doses required by the European Directive on Basic Safety Standards is commented on. Referrers and radiologists should be familiar with the dose quantities to inform patients about radiation risks and benefits. The application of effective dose on the cumulative doses from recurrent imaging procedures is also discussed.

Patient summary: Basic information on the measurement units (dosimetric quantities) used in medical imaging for reporting radiation doses should be understandable to patients. The Working Group on "Dosimetry for imaging in clinical practice" recommended that a brief explanation on the used dosimetric quantities and units included in the examination imaging report, should be available for patients. The use of the quantity "effective dose" to compare doses to which patients are exposed to from different radiological procedures and its uncertainties and limitations, should also be explained in plain language. This is also relevant for the dialog on to the cumulative doses from recurrent imaging procedures. The paper summarises these concepts, including the need to estimate the population doses required by the European Directive on Basic Safety Standards. Referrers and radiologists should be familiar with the dose quantities to inform patients about radiation risks and benefits.
\end{abstract}

Keywords: Dosimetric quantities, Diagnostic reference levels, Effective dose, Cumulative dose, Patient information

\section{Key points}

- Dosimetric quantities for reporting patient doses and comparing with diagnostic reference levels should be known by radiologists and radiographers.

- The radiation quantity "effective dose" with its uncertainties and limitations, may be used for comparison between different imaging modalities and procedures, and to explain the radiation risk to patients.

*Correspondence: eliseov@med.ucm.es

${ }^{1}$ Radiology Department, Complutense University, 28040 Madrid, Spain

Full list of author information is available at the end of the article
- The cumulative effective doses from recurrent imaging procedures in some groups of patients, may be considered in the justification and optimisation for the next imaging practices in these groups of patients.

\section{Introduction}

This review summarises the basic information on the dosimetric quantities used in medical imaging and interventional procedures for reporting patient doses 
and comparing doses delivered by different radiological procedures and different modalities. The estimate of the collective dose to the population (as required by the European regulation) [1] is also included. Patients should be informed on the benefits of diagnostic and interventional procedures but also on the radiation risks. Referrers, radiologists and radiographers should be familiar with the dose quantities. The proper use of the radiation protection quantity "effective dose" (ED), with its uncertainties and limitations, is summarised in the review. ED allows the relative risk of different radiological procedures and different modalities to be compared and it is also used for the estimation of collective doses (population doses) derived from medical imaging as required by the European Directive on Basic Safety Standards [1]. The application of effective dose on the cumulative doses from recurrent imaging procedures in certain groups of patients, is considered as additional information to help in some aspects of justification and optimisation.

This review has been produced by the EuroSafe Imaging working group (WG) on "Dosimetry for imaging in clinical practice", of the European Society of Radiology, to help understanding the patient dosimetry aspects in clinical imaging required by European and national regulations.

This is the content of the review:

1. Dosimetric quantities used in medical imaging and interventions for reporting patient doses and establishing diagnostic reference levels (DRLs).

2. Effective dose as a radiation protection quantity to compare different imaging modalities and to inform patients on the relative radiation risk.

3. Uncertainties and limitations on the use of effective dose in medical imaging.

4. Cumulative dose from recurrent imaging procedures and how this information may help to improve justification and optimisation.

5. Dosimetric information for practitioners, referrers and patients.

\section{Dosimetric quantities used in medical imaging for reporting patient doses (see Table 1)}

The dosimetric quantity Kerma (or Dose) Area Product $\left(\mathrm{P}_{\mathrm{KA}}\right.$ or DAP) is used for radiography, fluoroscopy and interventional procedures and takes into account the radiation produced by the $\mathrm{X}$-ray system and the irradiated area during the radiological procedure. It is usually measured in Gy.cm². In most X-ray systems (especially for interventional radiology systems) a second dosimetric quantity, called air kerma (or dose) at the patient entrance reference point (measured in $\mathrm{mGy}$ ) is also reported.

For projection radiography, in addition to $\mathrm{P}_{\mathrm{KA}}$, the entrance surface air kerma $\left(\mathrm{K}_{\mathrm{a}, \mathrm{e}}\right)$ (also known as entrance surface dose-ESD) is used, representing the radiation dose at the entrance of the patient, usually measured in mGy.

For Computed Tomography (CT), the Dose Length Product (DLP) is used. It takes into account the radiation produced by the scanner with the particular protocol of the examination and the scan range of the patient body examined. It is usually measured in mGy.cm.

$$
\operatorname{DLP}(\mathrm{mGy} . \mathrm{cm})=\mathrm{CTDI}_{\mathrm{vol}}(\mathrm{mGy}) \times \text { Scan Length }(\mathrm{cm})
$$

The Computed Tomography Dose Index (volume) $\left(\mathrm{CTDI}_{\mathrm{vol}}\right)$ (measured in mGy) is the parameter that best represents the average absorbed dose at a point within the scan volume for a particular scan protocol for a standardised phantom with 16 or $32 \mathrm{~cm}$ diameter [2].

The size-specific dose estimate (SSDE) can also be used for CT. SSDE considers corrections based on the size of the patient, using linear dimensions measured or determined from the patient or on patient images [3]. Dose estimates based on patient size are considered to be more accurate [4].

For mammography, the mean glandular dose is used, representing the mean absorbed dose in the glandular tissue of the breast, usually measured in mGy. Sometimes, the $\mathrm{K}_{\mathrm{a}, \mathrm{e}}$, measured in $\mathrm{mGy}$, is also reported [4].

In nuclear medicine, the radioactive activity (usually measured in $\mathrm{MBq}$ ) of the radiopharmaceutical administered to the patient is used.

Table 1 Quantities suitable for setting diagnostic reference levels (DRLs) ICRP-135 [4]

\begin{tabular}{|c|c|c|}
\hline Image modality & Recommended quantity & Recommended unit \\
\hline Radiography & Entrance-surface air kerma $\left(\mathrm{K}_{\mathrm{a}, \mathrm{e}}\right)$ or Kerma-Area Product $\left(\mathrm{P}_{\mathrm{KA}}\right)$ & mGy or mGy.cm² \\
\hline Mammography & Mean glandular dose $\left(D_{G}\right)$ or "average glandular dose" (AGD) and $K_{a, i}$ (incident air kerma) or $K_{a, e}$ & mGy \\
\hline Interventional fluoroscopy & kerma-Area Product $\left(P_{k A}\right)$ and air-kerma at the patient entrance reference point $\left(K_{a, r}\right)$ & Gy.cm² and $m G y$ \\
\hline Computed tomography & CTDI $I_{\text {vol }}$ (computed tomography dose index) and Dose Length Product (DLP) & mGy and mGy cm \\
\hline Nuclear medicine & Administered activity & $\mathrm{MBq}$ \\
\hline
\end{tabular}


It should be noted that the European Directive 2013/59/EURATOM on Basic Safety Standards [1] requires in the art. 58.b, that "Member States shall ensure that information relating to patient exposure forms part of the report of the medical radiological procedure".

\section{Dosimetric quantities used in medical imaging for establishing diagnostic reference levels (DRLs)}

A diagnostic reference level (DRL) is a form of investigation level used as a tool to aid in optimisation of protection in the medical exposure of patients for diagnostic and interventional procedures. It is used in medical imaging with ionising radiation, to indicate whether, in routine conditions, the amount of radiation used for a specified procedure (a clinical task) is unusually high or low for that procedure. For nuclear medicine, the administered activity (amount of radioactive material), or preferably the administered activity per unit of body weight, is used [4].

DRLs may be local, national or regional. The "local DRL" value is obtained from a few healthcare facilities, the "national DRLs" values are obtained from multiple facilities throughout a country and the "regional DRLs" values are obtained considering the national DRLs from a group of countries (a "region").

Median values of distributions of DRL values of dosimetric quantities at a facility should be compared with DRL values. But values of DRL quantities should not be used for individual patients because the DRL process is intended for optimisation of protection for groups of patients and is based on standard and not individual patients. For individual patients, the dosimetric values may be higher or lower than the DRL. The priority in medical imaging should always be the appropriate image quality or diagnostic information for the involved clinical task.

Table 1 summarises the ICRP recommended dosimetry quantities and units used in medical imaging to establish DRLs [4].

\section{Effective dose (ED) as a radiation protection quantity}

Effective dose was created by the ICRP to provide a dose quantity related to the probability of health detriment due to stochastic effects from exposure to low doses of ionizing radiation [5]. It is derived from the weighted sum of doses to tissues more sensitive to radiation and can only be derived by calculation. The tissue weighting factors are proposed by the ICRP and derived from epidemiological evidence. Effective dose is calculated for a "Reference Person" and not for an individual and is based on updated risk data and intended to apply as rounded values to a population of both sexes and all ages. Thus, for applications in medical imaging, the effective dose would be representative of a method and not individual (or even groups of) patients. Effective dose is not recommended for epidemiological evaluation $[5,6]$. It should be noted that radiation detriment is only a part of the total health detriment in medicine.

Effective dose is defined as the sum of the absorbed dose by organs and tissues weighted by factors representing the specific radiosensitivities of each organ/tissue. This quantity can be related with the increases of cancer and hereditary effects for standard persons. The following tissue weighting factors (Table 2) have been proposed by the IRCP [5]:

The age distributions for workers and the general population (for which the effective dose is derived) can be quite different from that of the overall age distribution for the population undergoing medical procedures using ionizing radiation and will also differ from one medical procedure to another depending on the age-and-sexprevalence of the individuals for the medical condition being evaluated [6].

\section{How to use effective dose to compare relative radiation risk from radiological procedures and imaging modalities}

The ICRP states that effective dose can be of practical value for comparing the relative doses related to stochastic effects in the following cases [5]:

Different diagnostic examinations and interventional procedures;

The use of similar technologies and procedures in different hospitals and countries;

The use of different technologies for the same medical examination, provided that the representative patients or patient populations for which the effective doses are derived are similar with regard to age and sex.

Table 2 Tissues and weighting factors proposed by the ICRP to estimate effective doses [5]

\begin{tabular}{llc}
\hline Tissue & $\mathbf{w}_{\mathbf{T}}$ & $\sum \mathbf{w}_{\mathbf{T}}$ \\
\hline $\begin{array}{l}\text { Bone-marrow (red), Colon, Lung, Stomach, Breast, } \\
\text { Remainder Tissues* }\end{array}$ & 0.12 & 0.72 \\
$\begin{array}{l}\text { (Nominal W applied to the average dose to 14 } \\
\quad \text { tissues) }\end{array}$ & 0.08 & 0.08 \\
$\begin{array}{l}\text { Gonads } \\
\text { Bladder, Oesophagus, Liver, Thyroid }\end{array}$ & 0.04 & 0.16 \\
Bone surface, Brain, Salivary glands, Skin & 0.01 & 0.04
\end{tabular}

* Remainder Tissues (14 in total): Adrenals, Extrathoracic (ET) region, Gall bladder, Heart, Kidneys, Lymphatic nodes, Muscle, Oral mucosa, Pancreas, Prostate, Small intestine, Spleen, Thymus, Uterus/cervix 
Table 3 shows some examples of effective doses from different imaging procedures from Germany [7] and the equivalent period of natural background radiation.

Effective dose may be used to compare doses from different imaging modalities. But it should be noted that for the same technology (e.g., fluoroscopy guided procedures) the distribution of doses inside the patient may be very different depending on the quality of the X-rays beam ( $\mathrm{kV}$ and filtration). For instance, the conversion factor to estimate effective dose from kerma area product (KAP) may increase in a $38 \%$ using high copper filtration in the $\mathrm{X}$-ay beam [8].

The ICRP has published a new document on the use of dose quantities in radiological protection, with a chapter on the use in medical exposures [9]. Several scientific papers have been published in the last years dealing with this topic [10-15].

Table 4 summarises some relevant aspects to take into account when using "effective dose" in medical imaging.

The new ICRP publication [9] suggests using the terminology that ED allows "approximate indicator of possible risk" with the additional consideration of variation in risk with age, sex and population group.
The amount of radiation and its distribution within the tissues of the body can be very different for several imaging modalities, even when a similar region of the body is being exposed. Since dose distributions from $\mathrm{x}$-ray and nuclear medicine procedures are very different, the effective dose is suitable for use in straightforward comparisons of doses from different techniques [9].

Effective dose is not the best quantity for making comparisons between doses for similar techniques applied in different departments or institutions. Modality-specific dose quantities (e.g., $\mathrm{P}_{\mathrm{KA}}$, DLP, CTDI ${ }_{\mathrm{vol}}$ ) should be used for this purpose. However, in circumstances in which the dose distributions within the body may be substantially different between procedures, effective dose may provide an appropriate measure for comparison [9].

A chest CT examination and a conventional chest $\mathrm{x}$-ray, both irradiate the lungs, but the effective dose from $\mathrm{CT}$ can be a few hundred times that of chest radiography, depending on the protocol technique (see Table 3). Referrers and practitioners should decide if such substantial difference in radiation dose is justified, for each individual patient.

Table 3 Typical effective doses and equivalent of natural background radiation time for different imaging procedures used in Germany [7]

\begin{tabular}{lll}
\hline Imaging procedure & Typical effective dose (ED) in (mSv) & $\begin{array}{l}\text { Approx. natural } \\
\text { background radiation (for } \\
\text { similar ED) }\end{array}$ \\
\hline Chest PA & & 3 days \\
Abdomen AP/PA & 0.02 & 2 months \\
CT-chest & 0.34 & 2.4 years \\
CT-abdomen and pelvis & 5.1 & 5.2 years \\
Coronary angiography & 11 & 1.5 years \\
Percutaneous coronary intervention (PCI) & 3.1 & 3 years \\
Endovascular aneurysm repair (EVAR) of the aorta & 6.4 & 8 years \\
Lung perfusion scintigraphy 160 MBq TC-99 m & 17 & 10 months \\
Positron emission tomography (oncology) & 1.8 & 2.2 years \\
(350 MBq F-18-FDG) & 4.6 &
\end{tabular}

The effective doses provided do not account for individual factors such as a patient's sex, age or constitution. The dose uncertainty may exceed a factor of 5 . Average natural radiation exposure in Germany: $2.1 \mathrm{mSv}$ per year

Table 4 Summary of the relevant aspects to take into account for the use of effective dose in medical imaging

\begin{tabular}{l}
\hline Relevant aspect \\
\hline Effective dose to "reference persons" can be used for comparing different imaging modalities \\
Useful for classifying different types of imaging procedures for communicating risks to clinicians and to patients \\
$\begin{array}{l}\text { Useful to inform decisions on justification of imaging procedures, planning research studies, and evaluation of unintended } \\
\text { exposures }\end{array}$ \\
$\begin{array}{l}\text { Uncertainties should be considered and variation in risk with age, sex and population group } \\
\text { Situations in which a single organ receives the majority of the dose Justification }\end{array}$ \\
\hline
\end{tabular}


The radiation risk of $1 \mathrm{mSv}$ is not the same for a child of 10 years, or an adult of 25 years or an adult of 70 years. And it is not the same for a man or for a woman.

Lifetime risk of cancer incidence per Sv may be around twice as great for the $0-9$ year's age at exposure group than for the 30-39 years group. For patients in their $60 \mathrm{~s}$, the lifetime risks from most imaging examinations are estimated to be about half those for patients in their $30 \mathrm{~s}$, falling to less than one-third for patients in their $70 \mathrm{~s}$ and about one-tenth for those in their $80 \mathrm{~s}$ [9].

\section{Estimation of collective doses from medical imaging}

The European Directive on Basic Safety Standards 2013/59/EURATOM [1] requires members of the European Union to make an estimation of population doses: "Member States shall ensure that the distribution of individual dose estimates from medical exposure for radiodiagnostic and interventional radiology purposes is determined, taking into consideration where appropriate the distribution by age and gender of the exposed". Note that it is required the distribution by age and gender of the exposed population.

Collective effective dose is not intended as a tool for epidemiological risk assessment, and it is inappropriate to use it in risk projections. The aggregation of very low individual doses over extended time periods is inappropriate, and in particular, the calculation of the number of cancer deaths based on collective effective doses from trivial individual doses should be avoided [5].
UNSCEAR (United Nations Scientific Committee on the Effects of Atomic Radiation) periodically requires different countries, in a survey on medical exposures, to provide the number of different diagnostic and interventional procedures with the used radiation doses. In these surveys it is also possible to include the effective dose estimations for the main groups of procedures. UNSCEAR also has a "User Manual" for the "Global survey of radiation exposure" [16] including the conversion factors to estimate effective doses from the dosimetric quantity reported by the X-ray imaging systems. Figure 1 shows a diagram on how to estimate effective doses from different imaging modalities using the conversion factors.

\section{Uncertainties and limitations for the use of effective dose}

The main uncertainties when trying to apply effective dose to individual patients are the intrinsic uncertainties derived from the Linear-Non-Threshold (LNT) model, when applied for diagnostic imaging, and for the risk quantification.

Uncertainties in both, effective dose calculation and radiation risk estimates, should be considered. The relative uncertainty in estimated values of ED for medical exposures for a reference patient is estimated to be about $40 \%$. The estimated risk of cancer may be a factor of three higher or lower when applied to a reference patient and will be more variable when applied to an individual [13, 17].

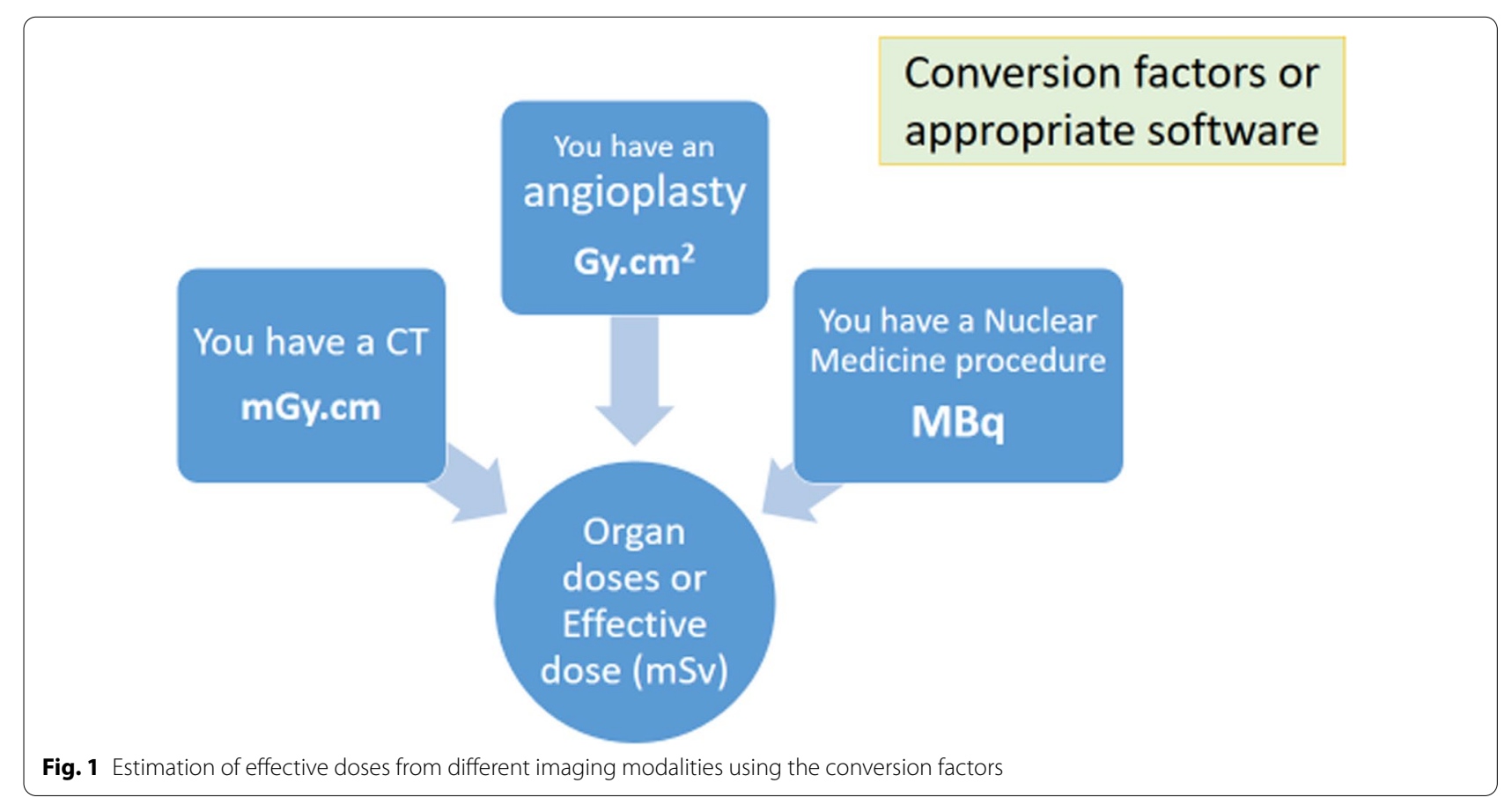


For nuclear medicine examinations, the uncertainties in the relative values of ED for a reference patient are of the order of $\pm 50 \%$. This is because of the tendency for doses to single organs (in particular the bladder) to be a substantial part of ED for some procedures. Prediction of risks of cancer for a reference patient derived using ED may be a factor of three higher or lower in practice [14].

The assessment and interpretation of effective dose from medical exposures of patients also needs to consider that some organs and tissues receive only partial exposures or a very heterogeneous exposure, which is the case especially with diagnostic and interventional procedures [5]. Stochastic risks estimations for individual organs may also be problematic for individual patients.

Stochastic risks are not modulated by dose rate, adaptive response, cellular repair of sub-lethal radiation damage, or genomic instability over long time periods (years) and ED applies only to an age-averaged, gender-averaged (male plus female) and region-averaged reference model [11].

In a recent paper on the "Past, present, and future of effective dose in Medicine", Martin et al. highlighted that ED is based on reference phantoms representing average individuals, but this is often forgotten in its application to medical exposures. The dose received by patients will differ from that assessed for reference phantoms, and the risk per Sv depend on the age and gender.

Newer techniques and the proliferation of phantoms of increasing sophistication are allowing for the refinement of calculations of more "patient-specific or size specific ED” using reference anatomical phantoms. But such values do not conform to the ICRP definition of ED, based on the reference phantoms, and need to be distinguished from the reference quantity [15].

\section{Cumulative dose from recurrent imaging procedures in some groups of patients and its limitations in practical use}

Several papers on recurrent imaging procedures with ionising radiation on the same patient have been published in the recent years [18-23]. Authors have focussed on patients undergoing recurrent $\mathrm{CT}$ exams that leads to cumulative effective dose (CED) of $\geq 100 \mathrm{mSv}$. Published data on the number of patients with cumulative effective doses $\geq 100 \mathrm{mSv}$ ranged from 0.6 to $3.4 \%$ in $\mathrm{CT}$ and around $4 \%$ in interventional radiology. Eighty per cent of patients having a CED of $\geq 100 \mathrm{mSv}$ had an oncological disease $[18,22]$.

The topic is relevant to improve justification and optimisation for the imaging procedures in the group of patients with high CED dose values. However, some authors [24] have alerted on several issues as if previous diagnostic radiation exposures should affect decisions on future examinations, concluding that bringing dose history into the decision process for justifying examinations may be not relevant for radiation risk and, rather than improving patient safety, would unnecessarily restrict access to radiation-based diagnostic examinations. In any way, the clinical context should be considered when highlighting the risks.

Getting 10 CTs during one hospital stay in a few days is different in radiation risk from getting $10 \mathrm{CTs}$ to follow disease over a period of 10 years. Cumulative doses from recurrent exposures may be useful information but converting these cumulative doses into radiation risks for individual patients should be avoided. The IAEA is preparing a "Joint Position Statement and Call for Action for strengthening radiation protection of patients undergoing recurrent radiological imaging procedures".

Dose management systems (DMS) may have a relevant role alerting referrers, radiologists and radiographers on previous examinations to profit from the existing diagnostic information and help to select the best imaging modality and protocol to be used in future [25]. Radiation doses may also be useful to inform patients on the benefits of the procedures and radiation risks, and on the potential need of a clinical follow up for interventional procedures if the skin doses may be near trigger levels [26, 27]. Refinements in the application of the justification criteria for these groups of patients should be considered.

One difficulty in many countries is the lack of a central data base for patient dose values and the difficulty to get the dose values from procedures carried out in different hospitals. Patient DMS may alert on cumulative high doses and this information should be available to the referrers and practitioners [28].

It should be noted that in the European Union, the directive 2013/59/EURATOM [1], requires individual optimisation, the evaluation of patient doses for some of the X-ray examinations and the capacity to transfer the information on the relevant parameters for assessing the patient dose, to the record of the examination. Information relating to patient exposure should be part of the report of the medical radiological procedures.

Sometimes referrers have little if any knowledge of patients' imaging histories, individual CED can be useful. With this information, radiologists, referrers and radiographers can make rational decisions regarding further imaging safely. Of course, critically ill patients, will never face delays or denials of CT studies in life-threatening situations [29]. The decision for any imaging procedure should always be taken by the qualified practitioner and the estimated cumulative dose (if available) should never be an impediment to perform an imaging procedure if it is clinically indicated. 
Cumulative dose may be used to refine, in some cases, the justification criteria (at the 3rd level, for individual patients) and the optimisation criteria for the next coming procedures to use low dose protocols if possible, and in some interventional procedures, using strategies to avoid skin radiation injuries. It is relevant to have alerts in the cumulative dose in the DMS but it is important to be aware that these systems are not always inter-connected between different hospitals in a city and country.

Personalised criteria for radiation protection in some patients (in parallel with the current approaches of the 'personalised medicine') should also be considered. The opinion of the patient needs to be taken into account. Some patients may accept additional radiation risks to confirm a diagnosis (e.g., a new CT). The result of this additional procedure may involve a relevant psychological benefit for the patient [30]. This may be considered as an ethical value concerning the patient autonomy to accept the radiation risk.

\section{Dosimetric information for practitioners, referrers, and patients}

Effective dose can be used for patients when explaining possible radiation risks. Comparisons with other sources of exposure, such as background radiation or the dose from cosmic rays during air travel may be useful in some cases. But quoting values for the risk to patients is not recommended as a general approach, both because of the uncertainties and the fact that this creates the impression that the risk is known accurately [15]. It could be said that a dose of $10 \mathrm{mSv}$ carries a nominal excess risk of $<1$ in 1000 , adding only slightly to the risk of developing cancer [15].

For patients: The suggested option for the Working Group on "Dosimetry for imaging in clinical practice" was to inform patients on "the dose values and units, reported by the X-ray system”. Other potential options, as informing on the effective doses derived from the imaging procedures or the estimation of the equivalent time in background radiation, were not considered appropriate at this step [27].

A brief explanation on the used dosimetric quantities and units included in the examination imaging report should be available for patients (e.g., what is a "dose area product" or a "dose length product" or the activity of a radionuclide).

If several imaging modalities may be used for a patient, effective dose may be used for comparison purposes but insisting that this quantity cannot be used to estimate individual radiation risk.

The proper information on radiation risk for patients (and its uncertainties) may be a critical issue to avoid unjustified patient fear. The individual health aspects should be part of this information considering the acceptability of the risk by the patient waiting for a good diagnosis [27].

For practitioners: More detailed information on dose values from the previous examinations may be useful to confirm the justification of the requested procedure, to confirm that the images and the reports are available and to decide the appropriate protocol (optimisation) for the new examination. This might be useful to consider non ionising radiation imaging modalities, namely MRI and Ultrasound in replacement of Ionising radiations, and this should be discussed with the referrers. For interventional procedures, the trigger levels need to be considered in the case of potential clinical follow-up. If new interventional procedures may be necessary, the details of the previous ones could help in avoiding potential skin radiation injuries.

For referrers: To also know the estimation of effective doses, or in some cases organ doses (e.g. mammography), may be of value to indicate which imaging modality to use depending on the diagnostic information to be obtained and previous examinations that have already taken place. Referrers should be aware of the benefits of MRI and Ultrasound, especially when they could replace Ionising radiation techniques. Estimations of effective doses (and the age and gender of the patients) may be useful to communicate the radiation risks and the benefits of new examinations. The uncertainties in all the dose estimations need to be considered.

\section{Abbreviations \\ AGD: Average glandular dose; AP: Anteroposterior; CED: Cumulative effective dose; CT: Computed Tomography; CTDlvol: Computed Tomography Dose Index (volume); DAP: Dose Area Product; $D_{G}$ : Glandular dose; DLP: Dose Length Product; DMS: Dose management systems; DRL: Diagnostic reference level; ED: Effective dose; ESD: Entrance surface dose; ET: Extrathoracic; EVAR: Endovascular aneurysm repair; Gy: Gray; ICRP: International Commission on Radiological Protection; Ka,e: Entrance surface air kerma; Ka,i: Incident air kerma; Ka,r: Air-kerma at the patient entrance reference point; KAP: Kerma area product; LNT: Linear-Non-Threshold; MBq: Megabecquerel; mGy: Milligray; PA: Posteroanterior; PCl: Percutaneous coronary intervention; $P_{\text {KA }}$ : Kerma area product; SSDE: Size-specific dose estimate; Sv: Sievert; WG: Working group.}

\section{Acknowledgements}

We thank Mr. Alistair Campbell from the European Society of Radiology (ESR) for the proofreading and editing of this manuscript. We thank Erik Briers for the review of the patient summary paragraph on behalf of the ESR Patient Advisory Group (ESR-PAG). This paper was reviewed and approved by the ESR Executive Council in April 2021.

\section{Authors' contributions}

Eliseo Vano (corresponding author) design of the paper, writing, implementing comments and producing final version. Guy Frija and Reinhard Loose, contribution to the paper design, comments and final approval. Graciano Paulo, Efstathios Efstathopoulos, Claudio Granata, \& Jonas Andersson, comments, refinements and final approval. All authors read and approved the final manuscript.

Funding

No funding received to produce this review. 


\section{Availability of data and materials}

All information contained in this review is from scientific papers, reports from international organisations (all referred in the references section) and opinions of the authors.

\section{Declarations}

Ethics approval and consent to participate

Not applicable.

\section{Consent for publication}

Not applicable.

\section{Competing interests}

The authors declare that they have no competing interests.

\section{Author details}

${ }^{1}$ Radiology Department, Complutense University, 28040 Madrid, Spain.

${ }^{2}$ Université de Paris, 12 Rue de l'École de Médecine, 75006 Paris, France. ${ }^{3}$ Institute of Medical Physics, Hospital Nuremberg, Prof.-Ernst-Nathan-Str. 1, 90419 Nuremberg, Germany. ${ }^{4}$ ESTESC-Coimbra Health School, Medical Imaging and Radiotherapy Department, Instituto Politécnico de Coimbra, Rua 5 de Outubro, S. Martinho Do Bispo, 3046-854 Coimbra, Portugal. ${ }^{5} 2 \mathrm{Nd}$ Department of Radiology, Medical Physics Unit, National and Kapodistrian University of Athens, Attikon University Hospital, 12462 Athens, Greece. ${ }^{6}$ Department of Paediatric Radiology, Institute for Maternal and Child Health - IRCCS "Burlo Garofolo", Trieste, Italy. ${ }^{7}$ Department of Radiation Sciences, Umea University, Umeå, Sweden.

Received: 15 June 2021 Accepted: 15 June 2021

Published online: 13 July 2021

\section{References}

1. European Council (2014) Council Directive 2013/59/Euratom of 5 December 2013, laying down basic safety standards for protection against the dangers arising from exposure to ionising radiation. Off J Eur Union L 13:73

2. Valentin J, International Commission on Radiation Protection (2007) Managing patient dose in multi-detector computed tomography (MDCT). ICRP Publication 102. Ann ICRP 37(1):1-79

3. AAPM. Size-specific Dose Estimates (SSDE) in Pediatric and Adult Body CT Examinations. AAPM Report No. 204 (2011). American Association of Physicists in Medicine, College Park, MD

4. Vañó E, Miller DL, Martin CJ et al (2017) ICRP Publication 135: diagnostic reference levels in medical imaging. Ann ICRP 46(1):1-144. https:// doi.org/10.1177/0146645317717209

5. (2007) The 2007 Recommendations of the International Commission on Radiological Protection. ICRP Publication 103. Ann ICRP $37(2-4): 1-332$

6. (2007) ICRP Publication 105. Radiation protection in medicine. Ann ICRP 37(6):1-63

7. SSK. Recommendations for medical imaging procedures (German Commission on Radiological Protection). Adopted at the 300th SSK meeting on 27 June 2019. https://www.ssk.de/SharedDocs/Beratungse rgebnisse E/2019/2019-06-270rientie e.html

8. Vano E, Sánchez RM, Fernández JM, Ten Jl (2019) Conversion factors to estimate effective doses from kerma area product in interventional cardiology. Impact of added filtration. Phys Med 68:104-111. https:// doi.org/10.1016/j.ejmp.2019.11.013

9. Harrison JD, Balonov M, Bochud F et al (2021) ICRP Publication 147: Use of Dose Quantities in Radiological Protection. Ann ICRP 50(1):9-82. https://doi.org/10.1177/0146645320911864
10. Borras C, Huda W, Orton CG (2010) Point/counterpoint. The use of effective dose for medical procedures is inappropriate. Med Phys 37(7):3497-3500. https://doi.org/10.1118/1.3377778

11. Fisher DR, Fahey FH (2017) Appropriate use of effective dose in radiation protection and risk assessment. Health Phys 113(2):102-109. https://doi.org/10.1097/HP.0000000000000674

12. Harrison JD, Balonov M, Martin CJ et al (2016) Use of effective dose. Ann ICRP 45(1 Suppl):215-224. https://doi.org/10.1177/0146645316 634566

13. Martin CJ (2008) The application of effective dose to medical exposures. Radiat Prot Dosimetry 128(1):1-4. https://doi.org/10.1093/rpd/ ncm425

14. Martin CJ (2011) Effective dose: practice, purpose and pitfalls for nuclear medicine. J Radiol Prot 31(2):205-219. https://doi.org/10.1088/ 0952-4746/31/2/001

15. Martin CJ, Harrison JD, Rehani MM (2020) Effective dose from radiation exposure in medicine: past, present, and future. Phys Med 79:87-92. https://doi.org/10.1016/j.ejmp.2020.10.020

16. UNSCEAR Global Survey on Medical Exposure. A User Manual. Draft Version May 2015. Accessed on 22 February 2021: http://www.survey. unscear.org/lib/exe/fetch.php?media=unscear_user_manual_version_ may2015.pdf

17. Durand DJ, Dixon RL, Morin RL (2012) Utilization strategies for cumulative dose estimates: a review and rational assessment. J Am Coll Radiol 9(7):480-485. https://doi.org/10.1016/j.jacr.2012.03.003 (PMID: 22748788)

18. Rehani MM, Hauptmann M (2020) Estimates of the number of patients with high cumulative doses through recurrent CT exams in 35 OECD countries. Phys Med 76:173-176. https://doi.org/10.1016/j.ejmp.2020. 07.014

19. Brambilla M, Vassileva J, Kuchcinska A, Rehani MM (2020) Multinational data on cumulative radiation exposure of patients from recurrent radiological procedures: call for action. Eur Radiol 30(5):2493-2501. https://doi.org/10.1007/s00330-019-06528-7

20. Rehani MM, Melick ER, Alvi RM et al (2020) Patients undergoing recurrent CT exams: assessment of patients with non-malignant diseases, reasons for imaging and imaging appropriateness. Eur Radiol 30(4):1839-1846. https://doi.org/10.1007/s00330-019-06551-8

21. Rehani MM, Yang K, Melick ER et al (2020) Patients undergoing recurrent CT scans: assessing the magnitude. Eur Radiol 30(4):1828-1836. https://doi.org/10.1007/s00330-019-06523-y

22. Frija G, Damilakis J, Paulo G, Loose L, Vano E, the European Society of Radiology (2021) Cumulative effective dose from recurrent CT examinations in Europe: proposal for clinical guidance based on an ESR EuroSafe imaging survey. Eur Radiol. https://doi.org/10.1007/ s00330-021-07696-1

23. Li X, Hirsch JA, Rehani MM, Ganguli S, Yang K, Liu B (2020) Radiation effective dose above 100 mSv from fluoroscopically guided intervention: frequency and patient medical condition. AJR Am J Roentgenol 215(2):433-440. https://doi.org/10.2214/AJR.19.22227

24. Walsh C, Murphy D (2014) Should the justification of medical exposures take account of radiation risks from previous examinations? $\mathrm{Br} J$ Radiol 87(1037):20130682. https://doi.org/10.1259/bjr.20130682

25. Loose RW, Vano E, Mildenberger P et al (2020) Radiation dose management systems-requirements and recommendations for users from the ESR EuroSafe Imaging initiative. Eur Radiol. https://doi.org/10.1007/ s00330-020-07290-x

26. Jaschke W, Bartal G, Martin CJ, Vano E (2020) Unintended and accidental exposures, significant dose events and trigger levels in interventional radiology. Cardiovasc Intervent Radiol 43(8):1114-1121. https:// doi.org/10.1007/s00270-020-02517-2

27. Vano E, Frija G, Stiller W et al (2020) Harmonisation of imaging dosimetry in clinical practice: practical approaches and guidance from the ESR EuroSafe Imaging initiative. Insights Imaging 11(1):54 
28. Loose RW, Vano E, Mildenberger P et al (2020) Radiation dose management systems-requirements and recommendations for users from the ESR EuroSafe Imaging initiative. Eur Radiol. https://doi.org/10.1007/ s00330-020-07290-x

29. Birnbaum S (2012) Cumulative dose estimates rationally used have a distinct role in patient management and care. J Am Coll Radiol 9(7):486-487. https://doi.org/10.1016/j.jacr.2012.04.006

30. Vano $E$ (2020) Recurrent imaging procedures with ionising radiation on the same patient. Should we pay more attention? J Radiol Prot 40(2):E14E17. https://doi.org/10.1088/1361-6498/ab7fbb

\section{Publisher's Note}

Springer Nature remains neutral with regard to jurisdictional claims in published maps and institutional affiliations.

\section{Submit your manuscript to a SpringerOpen ${ }^{\circ}$ journal and benefit from:}

- Convenient online submission

- Rigorous peer review

- Open access: articles freely available online

- High visibility within the field

- Retaining the copyright to your article

Submit your next manuscript at $\boldsymbol{\nabla}$ springeropen.com 